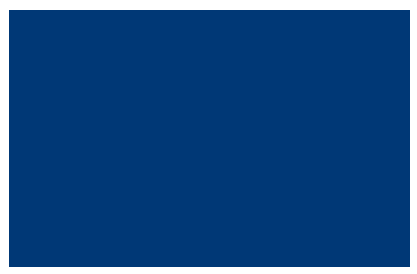

\title{
Crowns and other extra-coronal restorations: Introduction
}

\author{
R. W. Wassell ${ }^{1}$ J. G. Steele ${ }^{2}$ and A. W. G.Walls ${ }^{3}$
}

This series of articles is aimed at anybody who places crowns and other extra-coronal restorations (ie veneers and adhesive onlays or 'shims') on individual teeth. We hope that everyone from experienced practitioners to undergraduate students may find something of value. The aim of this series is to give guidance, based on available scientific evidence where possible, towards the provision of high quality restorations.

Treatment planning issues and materials choices feature at least as strongly as technique description.

We have concentrated on single tooth restorations, but all of the principles described also apply to more complex multiple restorations, including fixed bridges. However, we have not specifically covered replacement of missing teeth with bridges or implants. Replacement of teeth involves consideration of a range of additional issues and treatment planning decisions, whilst an entirely different set of technical rules are required for the consideration of implants. These will be left for other authors to address.

Where possible, we refer to published scientific evidence. Admittedly, randomly controlled clinical trials and systematic reviews are much scarcer in dentistry than in medicine. Consequently, much evidence is based on the experience of clinicians, or on laboratory or theoretical considerations. This does not necessarily devalue existing practices, but it does make it more difficult to make objective choices about treatment planning, materials or techniques. We have therefore tried to be honest when our advice stems solely from experience or theory.

The series consists of 13 individual articles, each of which should be able to stand alone. Each article will start with the specific learning objectives we hope to meet. In a series like this it is impossible to explore every technique or material ever described, this would make for very heavy reading and very dull writing. We hope though that these articles should enable the reader to evaluate his or her own practices against a set of fundamental principles.

\section{CONTENT OF THE SERIES}

A series of 13 Articles covering the following:

1. Changing patterns and the need for quality

2. Materials considerations

3. Pre-operative assessment

4. Endodontic considerations

5. Jaw registration and articulator selection

6. Aesthetic control

7. Cores for teeth with vital pulps

8. Preparations for full veneer crowns

9. Provisional restorations

10. Impression materials and technique

11. Try-in and cementation of crowns

12. Porcelain veneers

13. Resin bonded metal restorations 